\title{
Semiclassical Calculation of Diffractive Parton Densities
}

\author{
A. Hebecker \\ D.A.M.T.P., Cambridge University, Cambridge CB3 9EW, England
}

September 8, 2018

\begin{abstract}
In this talk the relation between the semiclassical approach and the concept of diffractive parton densities is discussed. The proton rest frame calculation is organized in a way that exhibits the hard partonic cross section and the diffractive parton density as the two fundamental ingredients. The latter one is a non-perturbative quantity which, in the present model, is explicitly given by integrals of non-Abelian eikonal factors in the colour background field.
\end{abstract}

\section{Introduction}

The presence of a large diffractive component of the small- $x$ deep-inelastic scattering structure functions [1] suggests an interesting interplay of soft and hard physics.

Consider the process of diffractive deep-inelastic scattering in the rest frame of the proton. In this frame a partonic fluctuation of the photon with virtuality $Q^{2}$ scatters off the proton, producing a final state with invariant mass $M$. In the case of a large transverse size of the fluctuation, corresponding at leading order to aligned jet configurations [2], a leading twist diffractive cross section arises. This can be seen explicitly from the semiclassical calculation, where the target proton is modelled by a classical colour field [3].

Hard diffraction can also be approached along the lines of inclusive deep-inelastic scattering, working in a frame where the incoming proton is fast. Focussing strictly on the leading power of $Q$, diffractive processes are then expected to allow a parametrization within the partonic approach, with diffractive parton distributions being the fundamental non-perturbative objects 近].

In the following the leading order derivation of the above partonic picture in the rest frame of the proton shall be outlined. More specifically, it will be shown how the semiclassical calculation can be factorized into hard and soft part and how a model for diffractive parton densities naturally arises from the soft part of the semiclassical calculation [5]. 


\section{Outline of the calculation in the scalar case}

The essential ideas of the calculation are most easily understood in a model containing scalar coloured particles. Consider the fluctuation of the incoming photon into a set of scalar partons which interact independently with the proton colour field (see Fig. 11).

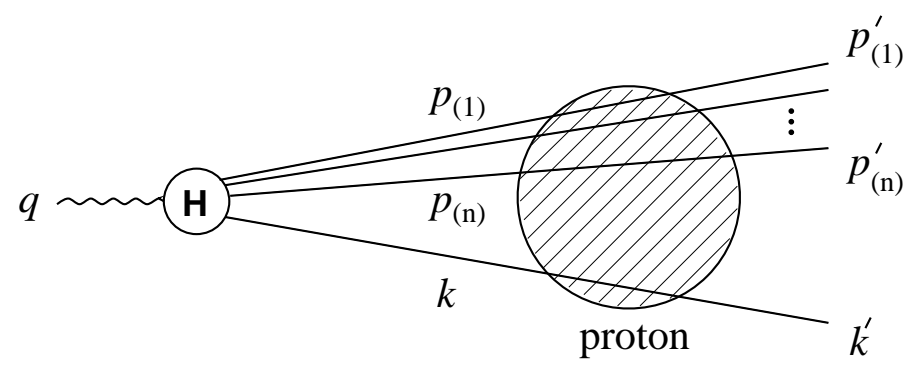

Figure 1: Hard diffractive process in the proton rest frame. The soft parton with momentum $k$ is responsible for the leading twist behaviour of the cross section.

Assume furthermore that the transverse momenta $p_{(j) \perp}^{\prime}(j=1 \ldots n)$ are hard, i.e. $\mathcal{O}(Q)$. A leading twist contribution to diffraction can arise only if the transverse momentum of the remaining parton is small, $k_{\perp}^{\prime} \sim \Lambda_{\mathrm{QCD}}$ [3].

The standard cross section formula for the scattering off a static external field reads

$$
d \sigma=\frac{1}{2 q_{0}}|T|^{2} 2 \pi \delta\left(q_{0}-q_{0}^{\prime}\right) d X^{(n+1)}, \quad \text { where } \quad q^{\prime}=k^{\prime}+\sum p_{(j)}^{\prime} .
$$

All momenta are given in the proton rest frame, $T$ is the amplitude corresponding to Fig. 1, and $d X^{(n+1)}$ is the usual phase space element for $n+1$ particles.

In the high energy limit each of the particles scatters off the field with an effective vertex proportional to $p_{0} \delta\left(p_{0}^{\prime}-p_{0}\right) \tilde{U}\left(p_{\perp}^{\prime}-p_{\perp}\right)$, where $\tilde{U}$ is the Fourier transform of the non-Abelian eikonal factor $U\left(x_{\perp}\right)$. This eikonal factor describes the colour rotation experienced by a parton penetrating the external field at transverse position $x_{\perp}$.

The resulting amplitude is given by

$$
\begin{gathered}
i 2 \pi \delta\left(q_{0}-q_{0}^{\prime}\right) T=\int T_{H} \prod_{j}\left(\frac{i}{p_{(j)}^{2}} 2 \pi \delta\left(p_{(j) 0}^{\prime}-p_{(j) 0}\right) 2 p_{(j) 0} \tilde{U}\left(p_{(j) \perp}^{\prime}-p_{(j) \perp}\right) \frac{d^{4} p_{(j)}}{(2 \pi)^{4}}\right) \\
\times\left(\frac{i}{k^{2}} 2 \pi \delta\left(k_{0}^{\prime}-k_{0}\right) 2 k_{0} \tilde{U}\left(k_{\perp}^{\prime}-k_{\perp}\right)\right),
\end{gathered}
$$

where $T_{H}$ stands for the hard part of the diagram in Fig. 1.

The integrations over the light-cone components $p_{(j)+}$ can be performed using the

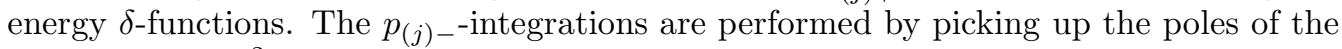
propagators $1 / p_{(j)}^{2}$. Since the external field is smooth and $T_{H}$ is dominated by the hard 
scale, $n-1$ of the $n$ transverse momentum integrations can be performed trivially. This is not the case for the last integration which will necessarily be sensitive to the small offshellness $k^{2}$. However, the $n-1$ performed integrations ensure that the eikonal factors associated with the high- $p_{\perp}$ partons are evaluated at the same transverse position. The resulting colour structure of the amplitude, after projection on a colour singlet final state, involves the trace of

$$
W_{x_{\perp}}\left(y_{\perp}\right)=\left(U\left(x_{\perp}\right)^{\dagger} U\left(x_{\perp}+y_{\perp}\right)-1\right)
$$

It is intuitively clear that only two eikonal factors are present since the high- $p_{\perp}$ partons are close together in transverse space. They are colour rotated like a single parton.

Under the further assumption that final state momenta of the high- $p_{\perp}$ partons are not resolved on the soft scale, the following result is derived,

$$
\frac{d \sigma}{d X^{(n+1)}}=\frac{k_{0}^{2}\left|T_{H}\right|^{2}}{\pi q_{0} N_{c}} \int_{x_{\perp}}\left|\int_{k_{\perp}} \frac{\operatorname{tr}\left[\tilde{W}_{x_{\perp}}\left(k_{\perp}^{\prime}-k_{\perp}\right)\right]}{k^{2}}\right|^{2} \delta^{2}\left(\sum p_{(j) \perp}\right) \delta\left(q_{0}-q_{0}^{\prime}\right) .
$$

In this expression the non-perturbative input encoded in the Fourier transform $\tilde{W}$ has totally decoupled from the hard momenta that dominate $T_{H}$.

\section{Results for scalar partons, quarks, and gluons}

The squared amplitude $\left|T_{H}\right|^{2}$ in Eq. (4) can be expressed through the partonic cross section $\hat{\sigma}(y)$ describing the collision of the photon, characterized by the standard variables $x$ and $Q^{2}$, with a parton carrying a fraction $y$ of the proton momentum. In Fig. 1 this corresponds to the interpretation of the line labelled by $k$ as an incoming line for the hard process. The cross section differential in $\xi=x\left(Q^{2}+M^{2}\right) / Q^{2}$ takes the form

$$
\frac{d \sigma}{d \xi}=\int_{x}^{\xi} d y \hat{\sigma}(y)\left(\frac{d f(y, \xi)}{d \xi}\right)
$$

Introducing the variable $b=y / \xi$ the diffractive parton density for scalars reads

$$
\left(\frac{d f(y, \xi)}{d \xi}\right)_{\text {scalar }}=\frac{1}{\xi^{2}}\left(\frac{b}{1-b}\right) \int \frac{d^{2} k_{\perp}^{\prime}\left(k_{\perp}^{\prime 2}\right)^{2}}{(2 \pi)^{4} N_{c}} \int_{x_{\perp}}\left|\int \frac{d^{2} k_{\perp}}{(2 \pi)^{2}} \frac{\operatorname{tr}\left[\tilde{W}_{x_{\perp}}\left(k_{\perp}^{\prime}-k_{\perp}\right)\right]}{k_{\perp}^{\prime 2} b+k_{\perp}^{2}(1-b)}\right|^{2} .
$$

Analogous considerations with spinor and vector partons result in the same factorizing result, but with a diffractive quark density

$$
\left(\frac{d f(y, \xi)}{d \xi}\right)_{\text {spinor }}=\frac{2}{\xi^{2}} \int \frac{d^{2} k_{\perp}^{\prime}\left(k_{\perp}^{\prime 2}\right)}{(2 \pi)^{4} N_{c}} \int_{x_{\perp}}\left|\int \frac{d^{2} k_{\perp}}{(2 \pi)^{2}} \frac{k_{\perp} \operatorname{tr}\left[\tilde{W}_{x_{\perp}}\left(k_{\perp}^{\prime}-k_{\perp}\right)\right]}{k_{\perp}^{\prime 2} b+k_{\perp}^{2}(1-b)}\right|^{2}
$$


or a diffractive gluon density

$$
\begin{aligned}
\left(\frac{d f(y, \xi)}{d \xi}\right)_{\text {vector }}= & \frac{1}{\xi^{2}}\left(\frac{b}{1-b}\right) \int \frac{d^{2} k_{\perp}^{\prime}\left(k_{\perp}^{\prime 2}\right)^{2}}{(2 \pi)^{4}\left(N_{c}^{2}-1\right)} \times \\
& \int_{x_{\perp}}\left|\int \frac{d^{2} k_{\perp}}{(2 \pi)^{2}}\left(\delta^{i j}+\frac{2 k_{\perp}^{i} k_{\perp}^{j}}{k_{\perp}^{\prime 2}}\left(\frac{1-b}{b}\right)\right) \frac{\operatorname{tr}\left[\tilde{W}_{x_{\perp}}^{\mathcal{A}}\left(k_{\perp}^{\prime}-k_{\perp}\right)\right]}{k_{\perp}^{\prime 2} b+k_{\perp}^{2}(1-b)}\right|^{2} .
\end{aligned}
$$

Here $W^{\mathcal{A}}$ is defined like $W$ but with the $U$ 's in the adjoint representation.

Note that the same kinematical picture arises if the soft interaction with the proton is modelled by two-gluon exchange [6]. While the kinematical factors associated with the different components of the photon wave function remain unchanged $\tilde{W}$ is substituted by a two-gluon form factor [7].

\section{Conclusions}

It has been shown that the semiclassical calculation in the proton rest frame is consistent with the concept of diffractive parton densities. The emerging physical picture is as follows. Leading twist diffraction arises from virtual photon fluctuations containing at least one low- $p_{\perp}$ parton. The interactions of the hard partons from the photon wave function with the proton colour field are kinematically irrelevant. In the Breit frame the elastic scattering of the low- $p_{\perp}$ parton off the field has to be reinterpreted as pair production by the fast proton. One parton from this colour singlet pair collides with the photon producing the above hard final state partons.

\section{References}

[1] M. Grothe (ZEUS), these proceedings; M. Dirkmann (H1), these proceedings

[2] J.D. Bjorken and J.B. Kogut, Phys. Rev. D8 (1973) 1341

[3] W. Buchmüller and A. Hebecker, Nucl. Phys. B476 (1996) 203; W. Buchmüller, M.F. McDermott and A. Hebecker, Nucl. Phys. B487 (1997) 283, erratum ibid.; W. Buchmüller, these proceedings

[4] G. Ingelman and P.E. Schlein, Phys. Lett. B152 (1985) 256; L. Trentadue and G. Veneziano, Phys. Lett. B323 (1994) 201; A. Berera and D.E. Soper, Phys. Rev. D50 (1994) 4328; A. Berera, these proceedings

[5] A. Hebecker, preprint DAMTP-97-10, hep-ph/9702373

[6] N.N. Nikolaev and B.G. Zakharov, J. Exp. Theor. Phys. 78 (1994) 598

[7] M. Wüsthoff, preprint ANL-HEP-PR-97-03, hep-ph/9702201; M. Wüsthoff, these proceedings 